\title{
Gastric Mucosal Phosphatidylcholine Hydroperoxide Increases during Cold Water-Immersion Restraint Stress in Rats
}

\author{
Wei-Ming Shian, Iwao Sasaki, Yasuhiko Kamiyama, \\ Hiroo Naito, Yuji Funayama, Seiki Matsuno and \\ Teruo Miyazawa* \\ The First Department of Surgery, Tohoku University School \\ of Medicine, Sendai 980-77, and *Department of Applied \\ Biological Chemistry, Faculty of Agriculture, Tohoku \\ University, Sendai 981
}

Shian, W.-M., Sasaki, I., Kamiyama, Y., Naito, H., Funayama, Y., Matsuno, S. and Miyazawa, T. Gastric Mucosal Phosphatidylcholine Hydroperoxide Increases during Cold Water-Immersion Restraint Stress in Rats. Tohoku J. Exp. Med., 1995, 176 (2), 127-130 — To know the role of lipid peroxidation in the pathogenesis of stress-induced gastric mucosal lesions, we performed the present study. Both gastric mucosal lesions and gastric mucosal phosphatidylcholine hydroperoxides $(\mathrm{PCOOH})$ levels during cold water-immersion restraint (CWIR) stress were measured by the chemiluminecence-HPLC method in Sprague-Dawley rats. Ulcer indices increased time-dependently during the stress. Gastric mucosal $\mathrm{PCOOH}$ levels also significantly increased and reached a peak level $(250 \%$ of untreated level) after $2 \mathrm{hr}$ of stress. These results suggest that lipid peroxidation may play an important role in the pathogenesis of gastric mucosal lesions induced CWIR stress. - phosphatidylcholine hydroperoxides $(\mathrm{PCOOH})$; cold water-immersion restraint stress; lipid peroxidation; gastric mucosal lesions; CLHPLC method

Oxygen-derived free radicals (oxyradicals) have been implicated in the pathogenesis of several experimental models of gastric ulceration. Cold water immersion plus restraint (CWIR) stress develops gastric corpus erosions in the rat. This effect depends upon the presence of intraluminal acid and high-amplitude, prolonged-duration contractions associated with diminished mucosal blood flow (Garrick et al. 1986a, b; Livingston et al.1988) which might result in cycles of ischemia and reperfusion. Although numerous studies have been undertaken to determine the relationship between gastric mucosal lesions and lipid peroxidation in CWIR stress, direct evidence for such a relationship is still lacking. In previous studies (Yoshikawa et al. 1986; Tarnasky et al. 1990), the link between gastric mucosal lesions and tissue lipid peroxidation could not be adequately assessed because the thiobarbituric acid (TBA) test commonly employed in such experiments is not specific for lipid peroxides. Miyazawa and colleagues $(1993,1994)$ have recently developed a new,

Received November 10, 1994; revision accepted for publication April 5, 1995.

Address for reprints: Dr. Iwao Sasaki, First Department of Surgery, Tohoku University School of Medicine, 1-1 Seiryomachi, Aoba-ku, Sendai 980-77, Japan. 
sensitive and selective assay for phospholipid hydroperoxides using high performance liquid chromatography (HPLC) combined with chemiluminescence (CL) detection. Using this CL-HPLC method, phospholipid hydroperoxides in human plasma and serum lipoproteins and in liver and brain of rodents were determined (Miyazawa et al. 1993).

In the present study, we investigated the changes in gastric mucosal phosphatidylcholine hydroperoxides (PCOOH) levels during CWIR stress in rats by the CL-HPLC method.

\section{Materials and Methods}

Seventy two male Sprague-Dawley rats, weighing around $200 \mathrm{~g}$, were used. After over 18-hr food deprivation, CWIR stress was performed according to Takagi and Okabe's method (1968). Each animal was tightly restrained inside a screen wire mesh, which was secured with staples. Animals were then immersed to the level of the xyphoid in water maintained at $22^{\circ} \mathrm{C}$. Before the stress and $30 \mathrm{~min}, 1,2,3$ and $4 \mathrm{hr}$ after the stress, animals were removed and sacrificed under ether anesthesia. The stomachs in 32 rats were harvested and opened along the greater curvature for assessment of lesions. In the remainder of the animals, the stomach was obtained immediately after laparotomy under ether anesthesia and gastric mucosa was scraped. Specimens were stored at $-80^{\circ} \mathrm{C}$ until the measurement of the $\mathrm{PCOOH}$ content.

Ulcer index was expressed by measuring the total length $(\mathrm{mm})$ of all lesions in the stomach induced during the stress. Gastric mucosal levels of $\mathrm{PCOOH}$ were measured by Miyazawa's method (1987).

Results are presented as the mean \pm s.e. Data were analyzed by the PLSD method of Stat-View (Abacus Concepts Inc., Berkeley, CA, USA). Differences at $p<0.05$ were defined as significant.

\section{Results}

\section{Ulcer index}

The ulcer indices are presented in Fig. 1. No macroscopic lesions were observed in untreated rats. Ulcer indices increased time-dependently during CWIR stress.

\section{Mucosal PCOOH levels}

In untreated rats, mucosal $\mathrm{PCOOH}$ level was $663.8 \pm 329.5 \mathrm{pmol} / 100 \mathrm{mg}$ protein. It gradually increased during stress and reached a value of $1903.3 \pm 408.3 \mathrm{pmol} / 100 \mathrm{mg}$ protein at 2-hr (Fig. 2). After the stress treatment, mucosal $\mathrm{PCOOH}$ levels were significantly elevated compared with untreated levels at all time points.

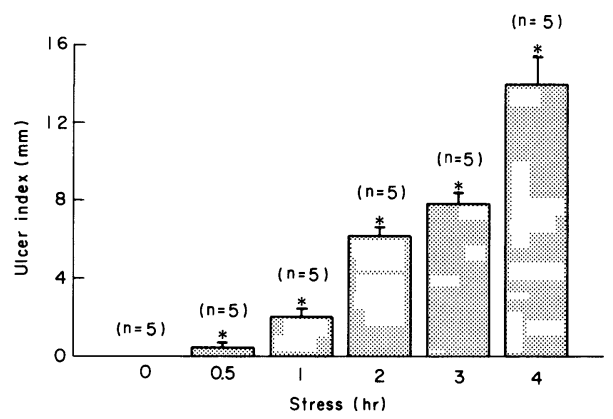

Fig. 1. Ulcer index induced by cold water-immersion restraint (CWIR) stress in rats.

( ) number of rats, values are expressed as mean \pm s.e.

*significant difference $(p<0.05$; vs. untreated rats) 


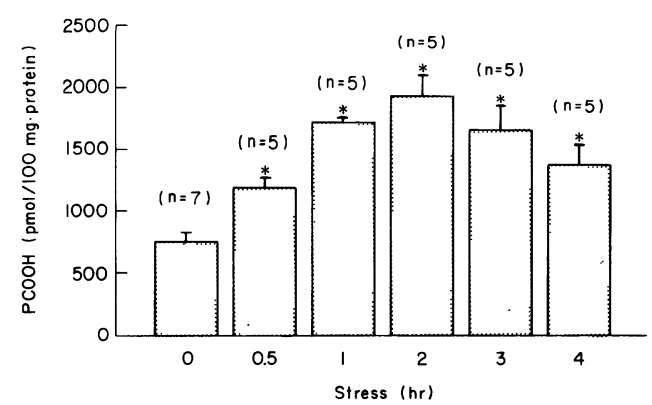

Fig. 2. Gastric mucosal $\mathrm{PCOOH}$ levels in cold water-immersion restraint (CWIR) stress in rats.

( ) number of rats, values are expressed as mean \pm s.e.

*significant difference $(p<0.05$; vs. untreated rats $)$

\section{Discussion}

The nature of the gastric mucosal barrier to chemical insult is not completely understood. One possible explanation for the efficient barrier function has been provided by Hills et al. (1983) and Schmitz and Renooij (1990) who proposed that the surface of the gastric mucosa is relatively hydrophobic, and thus may repel the aqueous gastric acid. The hydrophobic nature of the gastric mucosa is well documented; Wassef et al. (1979) reported that phosphatidylcholine constituted about half of the total phospholipids. Lichtenberger et al. (1983) reported that the gastric mucosal concentration of both phosphatidylcholine and phosphatidylethanolamine was increased around six times by administration of 16-16dimethyl prostaglandin $E_{2}$. They suggested that local accumulation of these phospholipids might be an integral component of the cytoprotective mechanism activated by prostaglandin treatment. Although there has been no report that phosphatidylcholine is more highly susceptible to peroxidation than other phospholipids in the gastric mucosa, one of possible means of damage to the gastric mucosa may be via oxidative degradation of mucosal barrier lipids.

It has been proposed that free radical-mediated lipid peroxidation may play an important role in the generation of acute gastric mucosal lesions in CWIR stress in rats. Yoshikawa et al. (1986) reported that TBA-reactive substances, an indirect index of lipid peroxidation, were significantly increased in gastric mucosa after CWIR stress in rats. They suggested that lipid peroxidation may play a role in the pathogenesis of gastric mucosal lesions. However, because measurement of TBA-reactive substance is not specific for lipid peroxidation, more exact method is necessary to verify their hypothesis. In the present study, we used a sensitive and selective assay method which measures phospholid hydroxides. The present results provide unequivocal evidence that the early phase of the CWIR stress is associated with a marked increase in $\mathrm{PCOOH}$ in gastric mucosa. These data support, but do not prove, the hypothesis that lipid peroxidative damage plays an important role in the development of CWIR stress-induced gastric mucosal lesions. Other pieces of evidence implicating oxidative damage after CWIR stress includes a decrease in reduced glutathion (a free radical scavenger ) content in both liver (Hirota et al. 1989) and gastric mucosa (Shian et al. 1994) after CWIR stress.

The precise role of lipid peroxidative damage in medicating the development of gastric mucosal lesions after CWIR stress is not clear. Although the present results and those of others (discussed above) demonstrate an association between lesion formation and the presence of indicators of lipid peroxidation, whether the relationship is a causal one is controversial. For example, Tarnasky et al. (1990) reported that inhibition of oxygen radical formation by chronic feeding of a sodium tungstate diet, oral administration 
allopurinopl, or intraperitoneal administration of dimethylsulfoxide did not significantly attenuated gastric lesions produced by CWIR stress in rats. The present study, for the first time, clearly indicated that CWIR stress produced peroxidative damage to gastric mucosal lipids. However, more studies will be required to define the precise role of lipid peroxidation in the pathogenesis of stress-induced gastric mucosal lesions.

\section{References}

1) Garrick, T., Buack, S. \& Bass, P. (1986a) Gastric motility is a major factor in cold restraint-induced lesion formation in rats. Am. J. Physiol., 250, G191-199.

2) Garrick, T., Leung, F.W., Buack, S., Hirabayashi, K. \& Guth, P. (1986b) Gastric motility is stimulated but overall blood flow is unaffected during cold restraint in the rat. Gastroenterology, 91, 141-148.

3) Hills, B.A., Butler, B.D. \& Lichtenberger, L. (1983) Gastric mucosal barrier: Hydrophobic lining to the lumen of stomach. Am. J. Physiol., 244, G561-568.

4) Hirota, M., Inoue, M., Ando, Y., Morino, Y., Sakamoto, K., Mori, K. \& Akagi, M. (1989) Role of hepatic glutathion turnover in the pathogenesis of stress-induced gastric mucosal lesion. Medical,Biochemical and Chemical Aspect of Free Radical. Proceedings of the 4th Biennial General Meeting of the Society for Free Radical Research, Kyoto, edited by O. Hayaishi, E. Niki, M. Kondo \& T. Yoshikawa, Elsevier Science Publishers, B.V. Amsterdam, pp. 1437-1440.

5) Lichtenberger, L.M., Graziani, L.A., Dial, E.J., Butler B.D. \& Hills, B.A. (1983) Role of surface-active phospholipids in gastric cytoprotection. Sience, 219, 13271329.

6) Livingston, E.H., Scremin, O.U., Yasue, N., Garric, T.R. \& Guth, P.H. (1988) Cold restraint produces foci of marked ischemia in the rat gastric corpus. Gastroenterology, 92, A266.

7) Miyazawa, T., Yasuda, K. \& Fujimoto, K. (1987) Chemiluminescence-high performance liquid chromatography of phosphatidylcholine hydroperoxide. Anal. Lett., 20, 915-925.

8) Miyazawa, T., Suzuki, T. \& Fujimoto, K. (1993) Age-dependent accumulation of phosphatidylcholine hydroperoxide in the brain and liver of the rat. Lipids, 28, 789-793.

9) Miyazawa, T., Lertsiri, S., Fujimoto, K. \& Oka, M. (1994) Luminol chemiluminescent determination of hydrogen peroxide at picomol levels using high-performance liquid chromatography with a cation-exchange resin gel column. J. Chromatogr., 667, 99-104.

10) Schmitz, M.G.J. \& Renooij, W. (1990) Phospholipids from rat, human, and canine gastric mucosa. Composition and metabolism of molecular classes of phosphatidylcholine. Gastroenterology, 99, 1292-1296.

11) Shian, W.M., Sasaki, I., Kamiyama, Y., Naito, H., Funayama, Y., Iwatsuki, A. \& Matsuno, S. (1994) Role of the glutathione in acute gastric mucosal lesion (AGML) induced by water-immersion-restraint stress (WIRS) in rats with obstructive jaundice. Ulcer Res., 21, 113-117. (in Japanese with English abstract)

12) Takagi, K. \& Okabe, S. (1968) The effect of drugs on the stress ulcer and recover processes of the stress ulcer. Jpn. J. Pharmacol., 18, 9-18.

13) Tarnasky, P.R., Livingston, E.H., Jacobs, K.M., Barbara, B., Zimmerman, J., Guth, P. \& Garric, T. (1990) Role of oxyradicals in cold water immersion restraintinduced gastric mucosal injury in the rat. Dig. Dis. Sci., 35, 173-177.

14) Wassef, M.K., Lin, Y.N. \& Horowitz, M.I. (1979) Molecular species of phosphatidylcholine from gastric mucosa. Biochim. Biophys. Acta, 573, 222-226.

15) Yoshikawa, T., Miyagawa, H., Yoshida, N., Sugino, S. \& Kondo, M. (1986) Increase in lipid peroxidation in rat gastric mucosal lesions induced by waterimmersion restraint stress. J. Clin. Biochem. Nutr., 1, 271-277. 